\title{
Colombian Applied Linguistics Journal
}

Indexed in:

Bases bibliográficas con comité de selección

(Sistemas de resúmenes)

Publindex Category A2

Education Research Abstracts (ERA), U.K.

Modern Language Association Bibliography (MLA), U.S.A.

Latindex

SciELO Colombia

Redalyc

Directories:

Ulrich's Periodicals

EBSCO Publishing

DOAJ

SHERPA/ ROMEO 
ISSN 0123-4641/ ISSNe 2248-7085

Vol.18 No. 2 July to December 2016

http://revistas.udistrital.edu.co/ojs/index.php/calj

Facultad de Ciencias y Educación

Maestría en Lingüística Aplicada a la

Enseñanza Del Inglés

Grupo de Investigación LECTROESCRINAUTAS

(C) Universidad Distrital Francisco José de Caldas 2016

\section{Editor}

Amparo Clavijo Olarte, Ph.D.

\section{Assistant to Editor}

Manuel Trujillo

\section{Scientific Committee 2016}

Patrick H. Smith, Ph.D.

University of Illinois at Urbana-Champaign, USA

Miguel Farias, Ph.D.

Universidad de Santiago de Chile, Chile

Theresa Austin, Ph.D.

University of Massachusetts Amherst, USA

Margaret Hawkins, Ph.D.

University of Winsconsin-Madison, USA

Gary Barkhuizen, Ph.D.

The University of Auckland, New Zealand

\section{Editorial Committee 2016}

Adriana González, Ph.D.

Universidad de Antioquia, Colombia

Judy Sharkey, Ph.D

University of New Hampshire, USA

Vander Viana, Ph.D.

University of Stirling, UK

Ángela María López-Velásquez,Ph.D.

Southern Connecticut State University, USA

Enric Llurda, Ph.D.

Universidad de Lleida, Spain.

\section{Proofreaders}

Reid Evans (English)

Jenny Jiménez Medina (Español)

Graphic cover designer

Nora Stella Torres

Designer

Julián Hernández - Taller de Diseño

\section{Impresión}

Javergraf

\section{Fondo de publicaciones \\ Universidad Distrital FRANCISCO JOSÉ DE CALDAS} CARRERA 24 no. 34-37

Tel. 3239300 Ext. 6203

Email: publicaciones@udistrital.edu.co

Contact: CALJ Editor, caljournal.ud@correo.udistrital.edu.co

Avda. Ciudad de Quito No. 64-81 Of. 704

Tel. 3238400 Ext. 6362

\section{Editorial Review Board}

Enric Llurda, Ph.D.

Universidad de Lleida, Spain.

Dora Calderón Ph.D.

Universidad Distrital Francisco José de Caldas, Bogotá, Colombia

Francisco Valdera Ph.D.

The University of Glasgow, Glasgow, United Kingdom

Sonia Casal MadinabeitiaPh.D

Universidad pablo de Olavide, Sevilla, Spain

Liliana Cuesta Ph.D

Universidad de La Sabana

Elisabeth Pladevall Ph.D.

Universitat Autònoma de Barcelona (UAB)

Miguel Farias, Ph.D.

Universidad de Santiago de Chile, Chile

Nelson Flores Ph.D

University of Pennsylvania, Pennsylvania, United States

Miao Li Ph.D

University of Toronto, Toronto, Canadá

Clara Inés Fonseca M.A.

Universidad de Cartagena, Cartagena, Colombia

Esperanza Vera M.A

Universidad Pedagógica Nacional, Bogotá Colombia

Melissa Fiori Ph.D.

Daemen Collegue, New York, United States

Mary Combs Ph.D.

University of Arizona, Arizona, United States

Maria Antonieta Celani Ph.D.

Universidad Católica de São Paulo, Brasil

Julia Renner Ph.D.

University of Vienna, Austria

Anna Ching-Shyang Ph.D.

Hsing-Wu Institute of Technology, Taipei, Taiwan

Jose David Herazo Rivera Ph.D.

Universidad de Córdoba, Colombia

Reza Dashtestani Ph.D.

University of Tehran, Iran

Rui Yuan Ph.D.

Chinese University of Hong Kong, China

Gloria Velez Rendon Ph.D.

Purdue University Calumet, United States

Nora Basurto Ph.D.

Universidad Veracruzana, Mexico

Moises Escudero Ph.D.

Universidad de Quintana Roo, México

Ludmila Marchenkova Ph.D.

Ohio University, United States

Peta Mathieu Ph.D.

Charles Sturt University, Australia

Alba Lucy Guerrero Ph.D.

Universidad de la Salle, Bogota, Colombia

Elizabeth Narváez Cardona Ph.D.

Universidad Autónoma de Occidente, Colombia

Michael Rodgers Ph.D.

Carleton University, Canada

Gerriet Janssen Ph.D.

Universidad de Los Andes, Colombia

Jhon Jairo Viáfara Ph.D.

Universidad Pedagógica y Tecnológica, Colombia

Deni Basaraba Ph.D.

Southern Methodist University, United States

Ana Pano Alaman Ph.D.

University of Bologna, Italy

José Aldemar Álvarez Ph.D.

Universidad del Valle, Cali, Colombia

\section{Creative Commons}

\section{(c) (1) $\Theta$}

This work is licensed under a Creative Commons Attribution-Non Commercial-No Derives 2.5 Colombia. Readers can read, copy and distribute the contets of this publication under the terms of Crative Commons, Colombia. For Further information refer to:

http://creativecommons.org/licenses/by-nc-nd/2.5/co 


\section{Declaración de buenas prácticas editoriales y normas éticas}

\begin{abstract}
"Este documento ha sido adaptado del documento para procedimientos y estándares éticos elaborado por Cambridge University Press, siguiendo las directrices para un buen comportamientoético en publicacionescientíficas seriadas del-Committee on Publication Ethics (COPE), International Committee of Medical Journal Editors (ICJME) y World Association of Medical Editors (WAME)"
\end{abstract}

\section{Responsabilidades de los editores}

Actuar de manera balanceada, objetiva y justa sin ningún tipo de discriminación sexual, religiosa, política, de origen, o ética de los autores, haciendo correcto uso de las directrices pronunciadas en la Constitución Política de Colombia en este aspecto.

Considerar, editar y publicar las contribuciones académicas únicamente por sus méritos académicos sin tomar en cuenta ningún tipo de influencia comercial o conflictodeinterés.

Acoger y seguir los procedimientos adecuados para resolver posibles quejaso malentendidos de carácter ético o de conflicto de interés. El editor y el comité editorial actuaran en concordancia con los reglamentos, políticas y procedimientos establecidos por la Universidad Distrital Francisco José de Caldas y particularmente en el Acuerdo 023 de junio 19 de 2012 del Consejo Académico, por el cual se reglamenta la política editorial de la Universidad y la normatividad vigente en el tema en Colombia. En todo caso se dará a los autores oportunidad para responder ante posibles conflictos de interés.

Cualquier tipo de queja debe ser sustentada con documentación y soportes que comprueben la conducta inadecuada.

\section{Responsabilidades de los revisores}

Contribuir de manera objetiva al proceso de evaluación de los manuscritos sometidos a consideración en la revista Colombian Applied Linguistics colaborando, en forma oportuna, con la mejora en la calidad científica deestos productosoriginales de investigación.

Mantener la confidencialidad de los datos suministrados por el editor, el comité editorial o los autores, haciendo correcto uso de dicha información por los medios que le sean provistos. No obstante, es su decisión conservar o copiar el manuscrito en el proceso de evaluación.
Informar al editor y al comité editorial, de manera oportuna, cuando el contenido de una contribución académica presente elementos de plagio o se asemeje sustancialmente a otros productos de investigación publicados o en proceso de publicación.

Informar cualquier posible conflicto de intereses con una contribución académica por relaciones financieras, institucionales, de colaboración o de otro tipo entre el revisor y los autores. Para tal caso, y si es necesario, retirar sus servicios en la evaluación del manuscrito.

\section{Responsabilidades de los autores}

Mantener soportes y registros precisos de los datos y análisis de datos relacionados con el manuscrito presentado a consideración de la revista. Cuando el editor o el comité editorial de la revista requieran esta información (por motivos razonables) los autores deberán suministrar o facilitar el acceso a esta. Al momento de ser requeridos, los datos originales entrarán en una cadena de custodia que asegure la confidencialidad y protección de la información por parte de la revista. Confirmar mediante una carta de originalidad (formato preestablecido por la revista) que la contribución académica so-metida a evaluación no está siendo considerada ò ha sido sometida y/ò aceptada en otra publicación. Cuando parte del contenido de esta contribución ha sido publicado o presentado en otro medio de difusión, los autores deberán reconocer y citar las respectivas fuentes y créditos académicos. Además, deberán presentar copia al editor y al comité editorial de cualquier publicación que pueda tener contenido superpuesto o estrechamente relacionado con la contribución sometida a consideración. Adicionalmente, el autor debe reconocer los respectivos créditos del material reproducido de otras fuentes. Aquellos elementos como tablas, figuras o patentes, que requieren un permiso especial para ser reproducidas deberán estar acompañados con una carta de aceptación de reproducción por parte de los poseedores de los derechos de autor del producto utilizado.

En aquellas investigaciones donde se experimente con animales se deben mantener y asegurar las prácticas adecuadas establecidas en las normas que regulan estas actividades.

Declarar cualquier posible conflicto de interés que pueda ejercer una influencia indebida en cualquier momento del pro-ceso de publicación. 
Revisar cuidadosamente las artes finales de la contribución, previamente a la publicación en la revista, informando sobre los errores que se puedan presentar y deban ser corregidos. En caso de encontrar errores significativos, una vez publicada la contribución académica, los autores deberán notificar oportunamente al editor y al comitéeditorial, cooperando posteriormentecon la revista en la publicación de una fe de erratas, apéndice, aviso, corrección, o en los casos donde se considere necesario retirar el manuscrito del número publicado.

CAL publicará cada dos años manuscritos de un mismo autor solamente si su contenido hace aportes diferentes en temática al del anterior proyecto de investigación.

\section{Responsabilidad de la Universidad Distrital}

La Universidad Distrital Francisco José de Caldas, en cuyo nombre se publica la revista Colombian Applied Linguistics y siguiendo lo estipulado en el Acuerdo 023 de junio 19 de 2012 del Consejo Académico, por el cual se reglamenta la Política

Editorial de la Universidad, se asegurará que las normas éticas y las buenas prácticas se cumplan a cabalidad.

\section{Procedimientos para tratar un comporta- miento no ético}

\section{Identificación de los comportamientos no éticos}

El comportamiento no ético por parte de los autores del cual tengan conocimiento o sea informada la revista, serán examinados en primera instancia por el Editor y el ComitéEditorial de la revista.

El comportamiento no ético puede incluir, pero no necesariamentelimitarse, a lo estipulado en la declaración de buenas prácticas y normas éticas de la revista Colombian Applied Linguistics, la reglamentación de la Facultad de Ciencias y Educación y la Universidad Distrital Francisco José de Caldas en esta materia.

La información sobre un comportamiento no ético, debe hacerse por escrito y estar acompañada con pruebas tangibles, fiables y suficientes para iniciar un proceso de investigación. Todas las denuncias deberán ser consideradas y tratadas de la misma manera, hasta que se adopte una decisión o conclusión exitosa.

La comunicación de un comportamiento no ético debe informarse en primera instancia preferiblemente al editor de la revista, en consecuencia al comité editorial o al comité de publicaciones de la Facultad de Ciencias y Educación. En aquellos casos donde los anteriores actores no den respuesta oportuna, deberá informarse el comportamiento no ético al comité de publicaciones de la Universidad Distrital Francisco José de Caldas.

La queja sobre un comportamiento no ético por partedelEditor oel ComitéEditorial dela revista deberá ser informada ante el comité de publicaciones de la Facultad de Ciencias y Educación de la Universidad Distrital.

\section{Investigación}

La primera decisión debe ser tomada por el editor, quien debeconsultar o buscar el asesoramiento del Comité Editorial, el Comité Científico y el Comité de Publicaciones de la Facultad de Ciencias y Educación, según el caso. Las evidencias de la investigación serán mantenidas en confidencialidad.

Un comportamiento no ético, que el Editor considere menor, puede ser tratado entre él y los autores sin necesidad de consultas adicionales. En todo caso, los autores deben tener la oportunidad de responder a las denuncias realizadas por comportamiento no ético.

Un comportamiento no ético de carácter grave se debe notificar a las entidades de afiliación institucional de los autores o que respaldan la investigación. El editor, en consideración con la Universidad Distrital, debe tomar la decisión de si debe o no involucrar a los patrocinadores, ya sea mediante el examen de la evidencia disponible o mediante nuevas consultas con un número limitado de expertos.

\section{Resultados}

(en orden creciente de gravedad, podrán aplicarse por separado o en combinación)

Informar a los autores o revisores donde parece haber un malentendido o mala práctica de las normas éticas.

Enviar una comunicación oficial dirigida a los autores o revisores que indique la falta de conducta ética y sirva como precedente para buen comportamiento en el futuro.

Hacer una notificación formal a los autores en la que se detalle la mala conducta con base en las evidencias del proceso de investigación. Informar a los comités editorial y científico de la revista sobre dicha conducta.

Retirar el artículo de la publicación de la revista y realizar una suspensión de cinco años al autor, periodo en el cual no podrá volver a publicar en la revista.

Denunciar el caso y el resultado de la investigación ante las autoridades competentes, en caso que el buen nombre de la Universidad Distrital se vea comprometido 